\title{
1993 Guidelines for the management of mild hypertension: Memorandum from a WHO/ISH meeting*
}

The present guidelines were prepared by the Guidelines Sub-Committee a of the WHO/ISH (International Society of Hypertension) Mild Hypertension Liaison Committee. They represent the third revision of the WHO/ISH guidelines and were finalized after discussions at the Sixth WHO/ISH Meeting on Mild Hypertension in Chantilly, France, on 28-31 March 1993. The new guidelines discuss the cardiovascular risk in patients with hypertension, the definition and classification of mild hypertension, drug treatment (including the elderly) and non-drug measures, cost-effectiveness, and further research.

\section{Introduction}

Patients with hypertension, even those with mild elevation of blood pressure, are at increased risk of cardiovascular disease, whether or not symptoms are present. In most countries, as many as $15-25 \%$ of the adult population are found at screening to have raised blood pressure; about two-thirds of them have mild elevation of blood pressure. However, the blood pressure is not persistently raised in all, and not all need to be treated with antihypertensive drugs.

In preparing these guidelines, the WHO/ISH Sub-Committee has been keenly aware that there are marked differences between individual patients with similar levels of hypertension, which have important implications for decisions about treatment. Hypertensive patients differ with respect to age, blood pressure elevation, organ damage and concomitant risk factors and diseases, and they live in societies where cardiovascular risk and economic resources also differ widely. Accordingly, the guidelines should not be rigid constraints to the practising doctor's decisions. Rather, they provide extensive, critical and well-balanced information on the benefits and limitations of the various diagnostic and therapeutic interventions, so that the physician may exert the most careful judgment in individual cases. Although the most reli-

\footnotetext{
* The previous WHO/ISH guidelines were published in the Butletin of the World Health Organization, 67: 493-498 (1989), and Journal of hypertension, 87: 689-693 (1989). The 1993 guidelines will be published simultaneously in Clinical and experimental hypertension. Requests for reprints should be sent to Chief, Cardiovascular Diseases, World Health Organization, 1211 Geneva 27, Switzerland. A French translation of this Memorandum will appear in a later issue of the Bulletin.

a Guidelines Sub-Committee: A. Zanchetti (Chairman), J. Chalmers (Convenor), K. Arakawa, I. Gyárfás, P. Hamet, L. Hansson, S. Julius, S. MacMahon, G. Mancia, J. Ménard, T. Omae, J. Reid and M. Safar.
}

Reprint No. 5409 able information is that provided by large randomized trials, these have their own limitations and not all aspects of the management of hypertension have been or can be determined by the results of randomized trials. Scientifically sound interpretation and cautious extrapolation of existing data can also be used to influence clinical decision-making.

\section{Highlights of 1993 guidelines}

New sections on:

- Systolic hypertension

- Hypertension in the elderly

- Assessment of cardiovascular risk

- Cost-effectiveness

- Issues needing further research

Update of sections on:

- Blood pressure measurements

- Diagnostic evaluation

- Goals of treatment

- Non-drug measures

- First-line drugs

- Correction of other major risk factors

\section{Cardiovascular risk}

\section{Assessment in patients with hypertension}

There is a continuum of cardiovascular risk associated with the level of blood pressure: the higher the blood pressure, the higher the risk of both stroke and coronary events (1). The dividing line between "normotension" and "hypertension" is arbitrary. The current definition is that this line is the level of blood pressure above which intervention has been shown to reduce the risk (2). It is well established that the 
lowering of even mildly elevated pressures reduces cardiovascular morbidity and mortality. However, a decision to intervene should not depend on blood pressure alone.

Indeed, among individuals with mild hypertension the risk of serious cardiovascular disease is also determined by a variety of factors other than the level of blood pressure. These include (see Table 1) increasing age, male gender, previous cardiovascular events, target organ damage (such as left ventricular hypertrophy or renal disease), smoking, diabetes, dyslipidaemia (high total and LDL-cholesterol with low HDL-cholesterol), central obesity, and a sedentary lifestyle (3). The presence of one or more of these factors may be a more important determinant of risk than a mild increase in the level of blood pressure. Since the absolute benefits of antihypertensive treatment will be determined by the absolute risk of cardiovascular disease (i.e., greater benefits among those at higher risk), each of these factors should be assessed prior to making decisions about treatment.

The absolute risk of serious cardiovascular disease varies greatly among individuals with mild hypertension. At one extreme, in elderly patients with a history of previous cardiovascular disease, at least three to five in every hundred will suffer a further serious event each year (4-7). At the other extreme, in young individuals with no other risk factors, fewer than one in every thousand will suffer a serious event each year $(8,9)$. While antihypertensive treatment will reduce risks in both these patient populations, it may take some decades for clinical benefits to become apparent in young patients at low initial risk.

Table 1: Cardiovascular risk factors favouring treatment

\section{Age $^{a}$}

Gender ${ }^{a}$

Family history of premature cardiovascular disease ${ }^{a}$

Raised systolic blood pressure

Raised diastolic blood pressure

Smoking

Raised total and LDL cholesterol

Reduced HDL cholesterol

Left ventricular hypertrophy

Previous cardiovascular events ${ }^{a}$

Previous cerebrovascular events ${ }^{a}$

Diabetes

Renal disease

Microalbuminuria

Obesity

Sedentary lifestyle

a Not modifiable.
The absolute risk of cardiovascular disease in mild hypertension may also vary substantially from one geographic region to another. Some of the regional variation in the risk of stroke and coronary events may be accounted for by regional differences in the prevalence of the risk factors listed above. However, other evidence indicates that there are some regional differences that cannot be accounted for by differences in established risk factors. Of particular note are the high rates of stroke in China and Russia. In these populations, stroke incidence is four times that in the USA and Western Europe but average population blood pressures are only slightly different. For this reason the treatment of mild hypertension in these populations may yield particularly large absolute benefits.

\section{Definition and classification}

\section{Blood pressure measurement}

Blood pressure is generally measured by the indirect method, using a mercury sphygmomanometer.

Before measurement commences, the patient should be seated for several minutes in a quiet room; the chair should provide comfortable back support. The arm muscles should be relaxed and the forearm supported with the cubital fossa at heart level (fourth intercostal space). Blood pressure may also be measured supine and standing, and in each position the arm should be supported at heart level. A cuff of suitable size is applied evenly to the exposed upper arm. Care should be taken to avoid tight sleeves. The "standard cuff" available in many countries may be too small. A cuff for adults must have a bladder $13-15 \mathrm{~cm}$ wide and $30-35 \mathrm{~cm}$ long so as to encircle the average arm. Larger cuffs are needed for fat arms and smaller ones for children. The cuff is rapidly inflated until the manometer reading is about $30 \mathrm{mmHg}$ above the level at which the pulse disappears, and then slowly deflated at approximately $2 \mathrm{mmHg} / \mathrm{second}$. During this time the Korotkoff sounds are auscultated through a stethoscope placed over the brachial artery.

The pressure at which the sounds are first heard is the systolic pressure. The diastolic pressure is the pressure at which the sounds disappear (phase V). Most of the major studies have used the latter point, i.e., disappearance of sounds; the use of muffling of sounds (phase IV), gives significantly higher diastolic pressure values, and is to be avoided. The systolic and diastolic pressures should be measured at least twice over a period of no less than 3 minutes; both should be recorded and the mean value for both should be used. It is also recommended that, on the first visit, the blood pressure should be measured on both arms. Measurement in the standing position 
should also be performed when postural hypotension is suspected and in the elderly in whom this condition may be more common.

"White coat hypertension" or "effect" is a condition in which blood pressure is elevated in the presence of a doctor but falls when the subject leaves the medical environment. Measurement by nurses or trained non-medical staff may reduce but not necessarily abolish the "white coat" effect (10). How the "white coat" effect can be precisely defined is controversial, however. Calculations on the basis of the difference between the blood pressure measured in the doctor's office and home blood pressure or daytime ambulatory blood pressure (see below) are questionable because this difference may depend on many more factors than just an alerting reaction. It is not known whether the "white coat" effect is an innocent phenomenon. Indeed, it has been suggested that subjects showing a marked difference between clinic and home blood-pressure measurements may be at higher cardiovascular risk; at least, they often have other concomitant risk factors (11). It is important, however, to recognize the condition in order to avoid unnecessary treatment in many subjects.

Semi-automatic and automatic devices for blood pressure measurement at home and for prolonged ( $\geq 24$ hours) ambulatory blood-pressure monitoring are now available (12). It should be stressed that all these devices should be tested for accuracy and reliability against the standard methods according to strict protocols (13). Ambulatory devices should be tested in ambulatory conditions. Home blood-pressure measurement has the advantage of providing many more readings in a quieter setting than blood pressure measurement in the doctor's office. Ambulatory blood pressure monitoring is an interesting research technique which is used to investigate blood pressure variability, behavioural influences on arterial pressure, and the time-course of the effects of antihypertensive therapy. It is also used, as are home blood-pressure readings, to provide a supplementary source of information for diagnostic and therapeutic decisions (12). Home and ambulatory blood-pressure values, however, cannot be equated to readings taken by the conventional method in the clinic by physicians or nurses. There is evidence, supported by a recent population survey (14), that both home bloodpressure and ambulatory blood-pressure values, averaged over 24 hours, are several $\mathrm{mmHg}$ lower than values measured in the clinic. Prognostic standards with regard to the level of blood pressure to be treated have been based on prospective studies relating casual or clinic blood pressure to morbidity and mortality. No prospective studies are available providing prognostically valuable standards for home or ambu- latory blood pressures. Therefore, it is likely that therapeutic decisions based on clinic blood pressure will differ from those based on home or ambulatory blood-pressure measurements (15). For the time being, the latter measurements should only be used in selected cases to complement the blood pressure values measured by the physician.

\section{Definition of mild hypertension based on diastolic blood pressure}

Diastolic blood pressure has generally been used to define mild hypertension. This choice is somewhat arbitrary, but is supported by the fact that diastolic blood pressure has been used as the criterion for inclusion in most randomized therapeutic trials, including those on mild hypertension (16).

Mild hypertension in adults can be defined as a persistent resting level of diastolic blood pressure (phase V) between 90 and $105 \mathrm{mmHg}$. "Borderline" hypertension (diastolic blood pressure $90-95 \mathrm{mmHg}$ ) accounts for about half of mild hypertensive patients. Community screening has shown that up to $20 \%$ of the population aged 50 years or above have diastolic pressures within the mild hypertensive range at the time of screening (17). With repeated measurements over periods of up to 3-6 months, the diastolic pressure of almost half of the subjects within the mild hypertension range falls to levels below this range $(18,19)$.

Persons whose resting values of diastolic blood pressure remain persistently at or above $90 \mathrm{mmHg}$ after repeated measurements are at increased risk of cardiovascular mortality and morbidity, and the risk clearly increases with the height of the diastolic blood pressure. Between $12 \%$ and $15 \%$ of such patients will develop moderate or severe hypertension (diastolic blood pressure $>105 \mathrm{mmHg}$ ) within 3-5 years (19), with a worse prognosis, while the remaining patients stay within the mild range. The risk of stroke is increased in patients with mild hypertension, and drug treatment has been clearly shown to reduce the risk by $35-40 \%$ (20). In populations with a high level of plasma lipids, the major cause of cardiovascular mortality and morbidity in patients with mild hypertension is usually ischaemic heart disease. Lowering of blood pressure with drugs also reduces the risk of non-fatal myocardial infarction and death from ischaemic heart disease by about 15-20\%, but the size of the benefit obtained with the treatments so far tested in controlled clinical trials appears to be slightly lower than that expected from a long-term decrease in blood pressure of the same magnitude (20).

In practice when the initial diastolic pressure averages between 90 and $105 \mathrm{mmHg}$, measurements 
Fig. 1. Definition and management of mild hypertension.

\section{DEFINITION:}

90-105 mmHg diastolic blood pressure (DBP) and/or

140-180 $\mathrm{mmHg}$ systolic blood pressure (SBP)

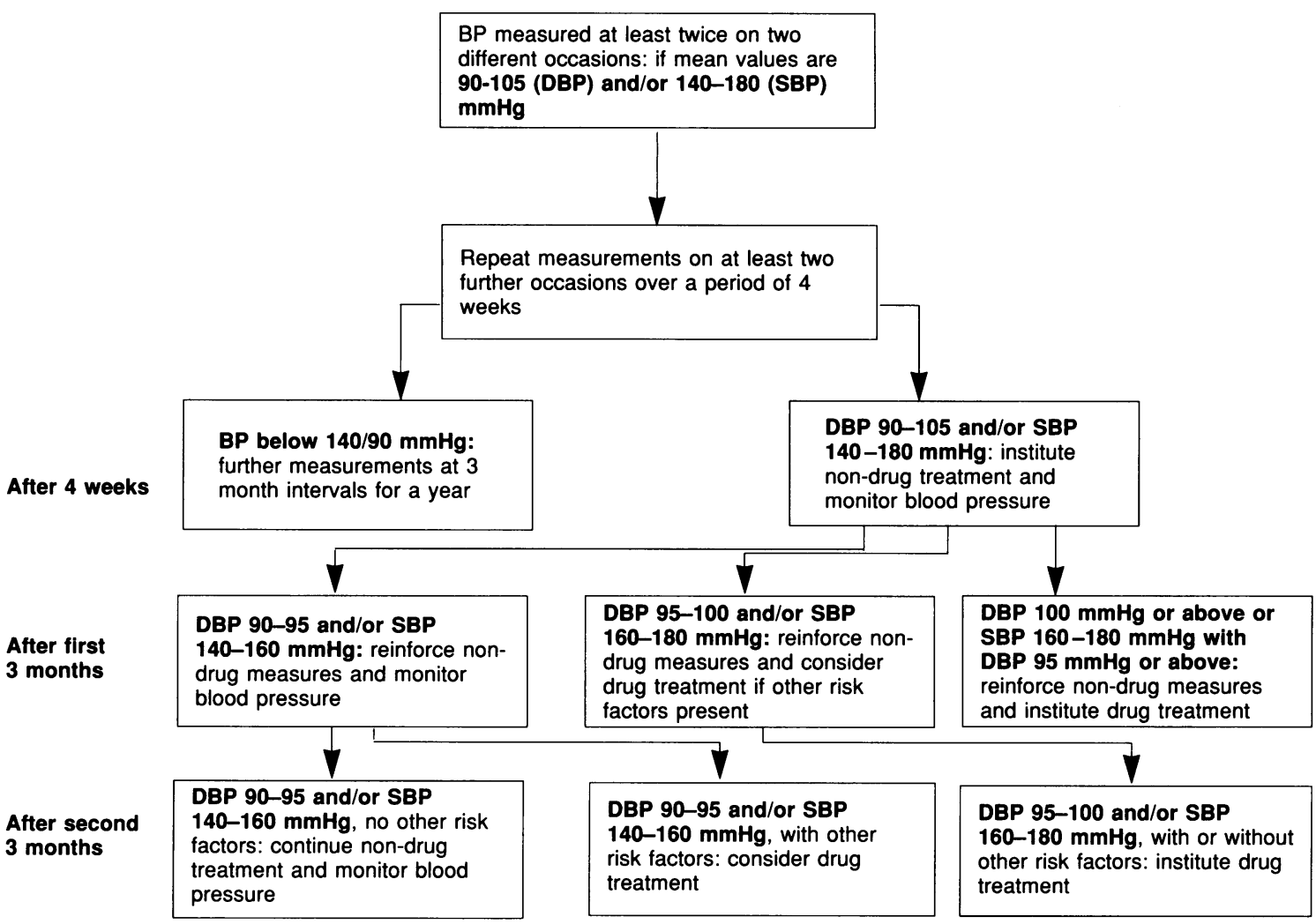

N.B. Institute drug treatment more promptly in patients with evidence of substantial risk of cardiovascular disease or in patients with blood pressure above the mild hypertension range.

should be repeated on at least two further occasions during the next 4 weeks. With repeated measurements both systolic and diastolic pressures often fall substantially. Before labelling a subject as hypertensive and deciding to initiate medical treatment, it is necessary, therefore, to identify those patients with a sustained high or increasing blood pressure.

All patients should be given advice to modify their lifestyle, as appropriate, by stopping smoking, reducing obesity, limiting alcohol and dietary saturated fat, and engaging in regular, mild dynamic exercise. This advice should form a very important part of the strategies to reduce blood pressure and to improve cardiovascular health. Salt restriction may assist in lowering the blood pressure. Management decisions should be made after discussing with the patient and his/her family and outlining the risks and benefits of various intervention strategies (21).

Practical guidelines are illustrated in Fig. 1.

\section{- At 4 weeks from initial measurements}

(1) Patients whose diastolic blood pressures fall below $90 \mathrm{mmHg}$ within 4 weeks should have further measurements at 3-month intervals for a year.

(2) If the diastolic blood pressure remains between 90 and $105 \mathrm{mmHg}$, appropriate non-drug treatment for all patients (see below) should be instituted and the blood pressure monitored on several occasions during the following 3 months. 
- After the first 3-month period

(3) If the diastolic blood pressure is $100 \mathrm{mmHg}$ or above, drug treatment should be instituted.

(4) If the diastolic blood pressure is $95-100 \mathrm{mmHg}$, non-drug measures should be reinforced, and drug treatment considered especially when other cardiovascular risk factors are present.

(5) If the diastolic blood pressure remains between 90 and $95 \mathrm{mmHg}$, non-drug measures should be reinforced and long-term observation of the patient continued.

\section{- After a second 3-month period}

(6) If the diastolic blood pressure is $95 \mathrm{mmHg}$ or above at the end of the second 3-month period, drug treatment should be instituted even when other cardiovascular risk factors are absent.

(7) Patients whose diastolic pressure remains between 90 and $95 \mathrm{mmHg}$ after prolonged observation also have an increased risk of cardiovascular disease. This is more marked when the systolic blood pressure is also elevated, as well as in elderly people, smokers, diabetics, individuals with raised plasma lipids or left ventricular hypertrophy, and those with a family history of cardiovascular disease. Drug treatment should be considered for such patients who are at higher risk. Lower-risk subjects, if not treated with drugs, should be further assessed at about 3month intervals and appropriate non-drug measures should be maintained or reinforced.

\section{Definition of mild hypertension based on systolic blood pressure}

Although most of the randomized therapeutic trials on mild hypertension have defined and treated patients on the basis of diastolic blood pressures only, there is mounting evidence that systolic values should also be taken into account in defining and managing mild hypertension. Indeed cardiovascular risk is as strongly associated with systolic as with diastolic values, with no evidence of a threshold below which a decrease in systolic pressure does not reduce the risk (22). Furthermore, some of the intervention studies on mild hypertension indicate that cardiovascular events more closely correlate with achieved systolic than diastolic blood pressure values (9).

From epidemiological data on incidence of strokes and coronary events, the range of systolic blood pressures corresponding to diastolic blood pressures of $90-105 \mathrm{mmHg}$, i.e., the range defining mild diastolic hypertension, is approximately 140-180 $\mathrm{mmHg}(22)$, and intervention trials have shown treatment benefits when systolic values of $160 \mathrm{mmHg}$ and above are lowered $(5,7)$.
When mild hypertension is defined according to systolic blood pressure, management should follow the same practical guidelines previously outlined for hypertension defined according to diastolic values. Fig. 1 summarizes a flow-chart of decision-making based on systolic as well as diastolic pressures. In summary, a diagnosis of mild hypertension based on systolic blood pressure can be made when systolic values of $140-180 \mathrm{mmHg}$ (associated or not with diastolic blood pressures of $90-105 \mathrm{mmHg}$ ) are repeatedly measured during at least 4 weeks, at which time only non-pharmacological intervention is to be recommended. After three additional months of observation, drug treatment should be instituted when the systolic blood pressure is $160-180 \mathrm{mmHg}$ and diastolic values are at least $95 \mathrm{mmHg}$, or with diastolic values below $95 \mathrm{mmHg}$ if other cardiovascular risk factors are present. After a second 3-month period, persistent systolic values of $160-180 \mathrm{mmHg}$ may justify drug treatment even when the diastolic blood pressure is below $95 \mathrm{mmHg}$ and there are no other risk factors; at this time even systolic values between 140 and $160 \mathrm{mmHg}$ with substantial risk factors may deserve drug treament.

In most cases both systolic and diastolic values will be in the respective mild hypertensive ranges, but in other cases either isolated mild diastolic hypertension or isolated mild systolic hypertension may occur. Isolated systolic hypertension can be found in adolescents and young people but is particularly common in the elderly $(6-10 \%$ of individuals aged 65-74 years in a recent population study (23)). Isolated systolic hypertension in adolescents and young people has different mechanisms compared with that of the elderly, and there is no evidence that isolated mild systolic hypertension in young people should be treated, apart from lifestyle counselling. On the other hand, isolated systolic hypertension in the elderly (often beyond the mild range) not only carries additional risk (22), but has recently been shown to benefit significantly from pharmacological reduction of elevated systolic blood pressure (see below). In adults below 60 years of age, although there is little direct evidence of benefit from treating isolated systolic hypertension (which is also infrequent at this age), it seems reasonable to consider drug therapy in subjects with systolic blood pressure values persistently equal to or above $160 \mathrm{mmHg}$ even if diastolic values are below $90 \mathrm{mmHg}$, and particularly when other risk factors are present.

\section{Classification of hypertension}

As mentioned at the beginning of this Memorandum, the risk associated with raised blood pressure increases progressively throughout the entire range of blood pressure values (1) and the dividing line 
between "normotension" and "hypertension" is arbitrary. However, the considerations made in the sections above allow an operational classification of hypertension to be made. This classification is illustrated in Table 2 as a practical guide to management. As previously mentioned, the term "mild" hypertension refers to individuals with either diastolic blood pressures in the range $90-105 \mathrm{mmHg}$ or systolic blood pressures in the range $140-180 \mathrm{mmHg}$. The term "borderline hypertension" is used in the subgroup of mild hypertensive subjects with diastolic blood pressure between 90 and $95 \mathrm{mmHg}$ or systolic blood pressure between 140 and $160 \mathrm{mmHg}$. The term "mild" is used to convey the information that the blood pressure is mildly elevated, but it does not imply that the absolute risk of cardiovascular disease is always mildly elevated. For instance, in an individual at high risk of stroke or myocardial infarction mild hypertension may considerably aggravate this risk, and the reduction of even mildly elevated blood pressure is likely to confer large benefits. The same considerations apply to patients with diabetic nephropathy. Furthermore, mild hypertension is highly prevalent and places a heavy burden on many populations.

Isolated systolic hypertension is a comprehensive term indicating all patients with systolic blood pressures equal to or greater than $140 \mathrm{mmHg}$ and diastolic blood pressures lower than $90 \mathrm{mmHg}$. This group with systolic pressures of $\geq 160 \mathrm{mmHg}$ have so far been shown to benefit from treatment; subjects with systolic blood pressure values between 140 and $160 \mathrm{mmHg}$ and diastolic pressures below $90 \mathrm{mmHg}$ can be classified as having "borderline" isolated systolic hypertension. Classifying subjects with diastolic blood pressures between 85 and $89 \mathrm{mmHg}$ or systolic blood pressures between 130 and $139 \mathrm{mmHg}$ as

Table 2: Classification of hypertension by blood pressure level

\begin{tabular}{lccc}
\hline & $\begin{array}{c}\text { Systolic } \\
\text { blood pressure } \\
(\mathrm{mmHg})\end{array}$ & $\begin{array}{c}\text { Diastolic } \\
\text { blood pressure } \\
(\mathrm{mmHg})\end{array}$ \\
\hline Normotension & $<140$ & and & $<90$ \\
$\begin{array}{l}\text { Mild hypertension } \\
\quad 140-180\end{array}$ & and/or & $90-105$ \\
$\begin{array}{l}\text { Subgroup: Borderline } \\
\text { hypertension }\end{array}$ & $140-160$ & and/or & $90-95$ \\
$\begin{array}{l}\text { Moderate and severe } \\
\text { hypertension }\end{array}$ & $\geq 180$ & and/or & $\geq 105$ \\
$\begin{array}{l}\text { Isolated systolic hyper- } \\
\text { tension (ISH) }\end{array}$ & $\geq 140$ & and & $<90$ \\
$\begin{array}{l}\text { Subgroup: Borderline } \\
\text { ISH }\end{array}$ & $140-160$ & and & $<90$ \\
\hline
\end{tabular}

a Risk to be indicated by reporting the actual values of systolic and diastolic pressures. "high normal" individuals (24) cannot be justified at the moment, and carries the risk of labelling a very large number of subjects.

As cardiovascular risk is associated with blood pressure levels in a continuous way, it appears reasonable that, beyond the range of mild hypertension, the risk of hypertension is indicated by reporting the actual values of systolic and diastolic blood pressure. As suggested in the 1978 WHO Expert Committee Report (25), the term "stage" is better used to indicate the absence, presence or severity of complications, rather than to classify different blood pressure levels (Table 3 ).

\section{Evaluation}

A complete history and physical examination are essential. Factors of significance include a family history of hypertension, diabetes, hyperlipidaemia, ischaemic heart disease or stroke; a personal history of symptoms suggestive of ischaemic heart disease, cardiac failure, or transient cerebral ischaemic episodes; a history of kidney disease, diabetes or bronchospasm; previous measurements of blood pressure; and details of lifestyle, educational level, and sociological factors. There should be careful quantitation

Table 3: Classification of hypertension by extent of organ damage

Stage I: $\quad$ No objective signs of organic changes

Stage II: At least one of the following signs of organ involvement :

- Left ventricular hypertrophy (X-ray, electrocardiography, echocardiography)

- Generalized and focal narrowing of the retinal arteries

- Proteinuria and/or slight elevation of plasma creatinine concentration $(1.2-2.0 \mathrm{mg} / \mathrm{dl})$

- Ultrasound or radiological evidence of atherosclerotic plaque (carotid arteries, aorta, iliac and femoral arteries)

Stage III: $\quad$ Both symptoms and signs have appeared as a result of organ damage. These include:

- Heart: angina pectoris, myocardial infarction, heart failure

- Brain: transient ischaemic attack, stroke, hypertensive encephalopathy

- Optic fundi: retinal haemorrhages and exudates with or without papilloedema

- Kidney: plasma creatinine concentration above $2.0 \mathrm{mg} / \mathrm{dl}$, renal failure

- Vessels: dissecting aneurysm, symptomatic arterial occlusive disease 
of smoking and alcohol consumption. Weight gain since early adult life can provide a useful index of excess body fat. Patients should be questioned on the ingestion of prohypertensive substances or drugs, notably oral contraceptives, nonsteroidal anti-inflammatory drugs, liquorice, cocaine, etc. Attention should be paid to the possible use of erythropoietin, ciclosporin, or steroids for concomitant diseases. Physical examination should include measurement of weight and height, evaluation of heart size, examination of optic fundi, and evidence of arterial disease in the carotid, renal and peripheral arteries. Certain minimum investigations should be performed in all patients. These are analysis for blood, protein, and glucose in the urine, microscopic examination of the urine, estimations of plasma potassium, creatinine, total and HDL-cholesterol, uric acid and blood glucose, and an electrocardiogram. Echocardiography is widely used in assessing left ventricular mass; the quality of the results depends on the patient's morphology and the operator's training, but its costeffectiveness in the evaluation of mild hypertensive subjects has not been investigated. None the less, the important prognostic role of left ventricular hypertrophy has made echocardiography a frequent component of the diagnostic work-up of the hypertensive patient, particularly in those cases in which evaluation of left ventricular mass and geometry may help to decide about initiation of treatment. Ultrasound is also useful in evaluating the carotid and abdominal aorta walls, and renal morphology. In selected cases, further investigations should be carried out to exclude curable causes of hypertension. The cost of investigations should be considered when a decision is made for a given patient.

\section{Treatment: general concepts}

\section{Effects of antihypertensive treatment on the risks of cardiovascular events}

Randomized controlled trials have shown that in patients with mild hypertension the lowering of blood pressure with antihypertensive drugs decreases morbidity and mortality from cardiovascular disease. On average, a 5-6 $\mathrm{mmHg}$ reduction in diastolic blood pressure (and $10 \mathrm{mmHg}$ reduction in systolic blood pressure) reduced stroke risk by about a third and the risk of coronary events by about a sixth (20). It is likely, however, that the benefits of antihypertensive therapy have been underestimated by most of the randomized controlled trials for at least three reasons: (1) in many trials of active vs placebo treatment there has been extensive cross-over of patients from placebo to active treatment; (2) in several trials low-risk patients were preferentially included; and
(3) most trials have been relatively short term ( 3 to 5 years' duration), and the full effects of blood pressure reduction, on coronary events in particular, may take a decade or more to become manifest. Since the absolute risk of coronary events in young patients is low, the initial goal of treatment is to prevent the progression of disease processes such as left ventricular hypertrophy and, possibly, atherosclerosis rather than discrete events (26).

\section{Factors influencing initiation of treatment}

Diastolic and systolic blood pressure values are important for initiation of treatment, and it should be stressed that, whenever blood pressure values above the mild hypertension range (i.e., systolic 180 and diastolic $105 \mathrm{mmHg}$ or above; see Table 2) are measured, a decision to treat with drugs should be taken after a shorter observation period than the one suggested in Fig. 1 for mild hypertension.

However, several factors other than diastolic and systolic blood pressures and age may influence the decision to begin drug treatment.

(1) Gender. Premenopausal women are at a $50 \%$ lower risk of cardiovascular disease than men of the same age (3), and the absolute benefit of treatment is less conspicuous in this group of women than in men, unless other risk factors are present. Furthermore, in males hypertension appears to be frequently underdiagnosed and undertreated (23).

(2) Cardiovascular complications. Clinical, echocardiographic or radiological evidence of left ventricular hypertrophy is a predictor of a substantially higher incidence of morbid events $(27,28)$ and is therefore a clear indication to begin treatment. Clinical, electrocardiographic or angiographic evidence of ischaemic heart disease in hypertensive patients requires lowering of blood pressure in addition to treatment of the underlying disorder. A history of cerebrovascular disease (e.g., transient cerebral ischaemia or stroke, especially cerebral or subarachnoid haemorrhage) is also a clear indicator for the initiation of treatment. It has not been fully proven, however, that antihypertensive therapy can prevent the recurrence of strokes. The presence and nature of cardiovascular complications may also influence the choice of treatment.

(3) Renal disease. Raised serum creatinine and proteinuria are predictors not only of renal impairment but also of a higher incidence of cardiovascular events (29). If either is present, drug treatment should be started. In patients with chronic renal failure, particularly those with diabetic nephropathy, even blood pressure values in the borderline range 
represent a considerable risk and must be lowered by drugs.

(4) Diabetes. Diabetes and hypertension are frequent concomitant diseases and hypertension is a potent additional risk factor in diabetes and vice versa. The presence of microalbuminuria is an early indicator of this increase in risk (30). There is good evidence that treatment of high blood pressure or even a lowering of normal blood pressure in diabetic patients reduces microalbuminuria, slows the decline in renal function, and delays the development of diabetic nephropathy (31). Therefore, the presence of diabetes (both insulin- and non-insulin dependent forms) in patients with mild hypertension is a clear indication to begin antihypertensive treatment. In these patients particular attention should also be paid to accompanying disorders of lipid metabolism.

(5) Other cardiovascular risk factors. Continued cigarette smoking, elevated fasting glucose, elevated serum total cholesterol, and low HDL-cholesterol all markedly increase the cardiovascular risk associated with high blood pressure (3) and increase the absolute benefit of lowering the blood pressure. Therefore, the presence of one or more of these risks should influence the decision towards early drug treatment and the intensive use of appropriate nondrug measures, and may also influence the choice of the antihypertensive drugs.

(6) Family history. A family history of hypertension or of premature stroke, heart disease, or sudden cardiac death should influence the decision towards early drug treatment.

(7) Low-income populations. A decision to initiate drug treatment and, more broadly, the range of actions desirable for hypertension control will vary with a population's resources, constraints, mortality structure, and the resulting health care priorities. In developing countries costs may make it difficult to extend all choices of diagnostic procedures and drug therapies to all individuals with mild hypertension. Advice on modification of lifestyle should be of particular benefit in such circumstances before resorting to drug therapy. Although individualized counselling and structured programmes (see below) will be difficult to provide in most developing countries, population-based initiatives to reduce average blood pressure levels would be worthwhile. In low-income societies it is a realistic approach to train auxiliaries for measuring blood pressure, and for simple urine testing, and to follow a less rigorous evaluation schedule. Although higher-risk individuals should be treated pharmacologically, careful choice of drugs with increased emphasis on cost-effectiveness becomes particularly important with growing economic constraints. Increasing the public's awareness of the hazards of hypertension, measuring of blood pressure at every opportunity, regular surveillance of hypertensive individuals, and educating the population about factors that may raise blood pressure or aggravate existing hypertension are measures as important in low income as in affluent populations. Political and public education is particularly important in reducing tobacco dependency, and promoting the production and marketing of healthy foods. Indeed, it is the responsibility of the medical practitioner to participate in educating the public and in persuading governments.

\section{Hypertension in the elderly}

In absolute terms, hypertension is a much greater risk of cardiovascular events in the elderly than in younger people. In Western populations, among those with mild hypertension the 10-year risk of a major cardiovascular event ranges from less than $1 \%$ in individuals aged 25-34 years to more that $30 \%$ in those aged 65-74 years (3). Correspondingly, numerous intervention trials have shown that the absolute benefit of antihypertensive therapy is particularly high in the elderly.

In addition to the results of the European Working Party on High Blood Pressure in the Elderly (EWPHE) trial published in 1985 (4), which clearly demonstrated the beneficial effect of antihypertensive medication in the elderly, the results of three prospective placebo-controlled therapeutic trials in elderly hypertensives have been reported in 1991 and 1992. All demonstrated significant reduction of cardiovascular morbidity or mortality. The Systolic Hypertension in the Elderly Program (SHEP) (5) specifically examined the value of antihypertensive treatment in elderly men and women ( $\geq 60$ years) with isolated systolic hypertension (systolic blood pressure $160-219 \mathrm{mmHg}$ and diastolic blood pressure $<90 \mathrm{mmHg}$ ): highly significant reductions in fatal and non-fatal cardiovascular events were observed. The Swedish Trial in Old Patients with Hypertension (STOP-Hypertension) (6) evaluated active antihypertensive therapy versus placebo in "old elderly" patients (aged 70-84 years) with systolic blood pressure $180-230 \mathrm{mmHg}$. Fatal and nonfatal strokes were reduced by $47 \%$, all "primary endpoints" (i.e., fatal and non-fatal stroke, fatal and non-fatal myocardial infarction and other cardiovascular death) by $40 \%$ and total mortality by $43 \%$, all changes being statistically highly significant. The Medical Research Council trial in older adults (aged 65-74 years) (7) with systolic blood pressure of $160-209 \mathrm{mmHg}$ and a diastolic blood pressure $<115 \mathrm{mmHg}$ showed a $25 \%$ reduction in stroke mor- 
tality and morbidity and a $17 \%$ reduction in all cardiovascular events, both of which were significant. From the results of all these trials it is obvious that antihypertensive therapy in elderly patients with hypertension, be it isolated systolic or combined systolic and diastolic, provides relative benefits, in terms of percent reduction in cardiovascular morbidity and mortality, that are of at least the same magnitude (about $20-50 \%$ ) as the benefits of treatment in young and middle-aged hypertensive patients. Furthermore, since the cardiovascular event incidence is high in the elderly, the same relative reduction in risk as in younger patients provides a greater absolute benefit in this age group.

In all trials, with the single exception of the Medical Research Council trial, benefit ranges between one event prevented each year for every 35 treated patients and one event prevented for every 90 treated patients. Old age, therefore, renders antihypertensive therapy particularly beneficial. According to the STOP-Hypertension study (6), significant benefits are also seen in the "old elderly" without any clear upper limit of age. In the oldest patients, however, caution is needed in the use of drugs, especially if other medications are also prescribed for associated diseases. It should also be remembered that in elderly patients the blood pressure elevation can often be controlled by low-dose medication. Bearing in mind these precautions, antihypertensive therapy can be instituted in the elderly along the same general guidelines as in young and middle-aged patients.

\section{Goal of treatment}

Since the relationship between blood pressure and cardiovascular risk is continuous, it seems appropriate for the goal of treatment to be the maximum tolerated blood pressure reduction. There is very good evidence from epidemiological studies that, within the "normal" range of both systolic and diastolic pressures, the lower the blood pressure, the lower the risks of both stroke and coronary events (1). The claim that lowering the diastolic pressure below $85 \mathrm{mmHg}$, at least in particular groups of patients (such as those with ischaemic heart disease), raises the risk above that associated with a more moderate blood pressure reduction (32) is unproven, but is currently being tested by a randomized trial (33). Until such evidence becomes available, it may be worth recalling that the safety of blood pressure reduction even at very low initial blood pressure levels has been indicated by the results of trials in patients with congestive heart failure or after myocardial infarction who have been treated with angiotensin-converting enzyme (ACE) inhibitors or beta-blockers. Furthermore, patients in the SHEP study (5), with an aver- age initial diastolic blood pressure of $77 \mathrm{mmHg}$, exhibited substantial benefits with further lowering of diastolic blood pressure.

On the basis of available evidence, it would seem desirable to achieve blood pressures of the order of at least $120-130 / 80 \mathrm{mmHg}$ in young patients with mild hypertension. In elderly patients with elevation of both systolic and diastolic blood pressure it would seem desirable to lower blood pressure to below $140 / 90 \mathrm{mmHg}$, while in patients with isolated systolic hypertension the goal of treatment should be to achieve a systolic blood pressure of at least $140 \mathrm{mmHg}$ if this is tolerated. When home blood pressure or ambulatory blood pressure measurements are used to help in the evaluation of blood pressure achieved by treatment, it should be remembered that the values provided by these methods are, on average, several $\mathrm{mmHg}$ lower than the clinic blood pressures (14); therefore, the blood pressure to be attained by treatment, when assessed by these techniques, should be set at a lower level to avoid undertreatment.

\section{Modes of treatment Non-pharmacological interventions}

Several non-pharmacological interventions are recommended in the primary prevention of hypertension and other cardiovascular diseases and have been the object of a recent joint WHO/ISH statement (21). These interventions have also been shown to lower blood pressure in patients with mild hypertension. As discussed in this statement $(21)$, weight reduction in overweight subjects, reduction of alcohol consumption to no more than $20-30 \mathrm{~g}$ of ethanol per day, regular mild (not strenuous) exercise in sedentary subjects (such as walking, jogging, cycling or swimming), and sodium chloride restriction to no more than $5 \mathrm{~g}$ /day (at least in some patients) are effective in lowering the blood pressure. It is known, however, that these lifestyle modifications are difficult to apply on a large scale, that compliance to such recommendations is poor in the long-term, and that the ability of non-pharmacological interventions to reduce mortality and morbidity in hypertension has not been proved directly. Nevertheless, it appears reasonable to advise that efforts to lower blood pressure by lifestyle modifications should normally precede any decision about the necessity for drug treatment of mild hypertension. It should be remembered that some of these measures may take several months to become fully effective. If a decision is made to begin drug treatment, a structured programme of non-pharmacological intervention remains an important component of the overall therapeutic pro- 
gramme, even in patients with an initial blood pressure above the mild hypertension range, for whom drug requirements may consequently be reduced.

\section{Control of associated risks}

Tobacco. All the large-scale trials of treatment of mild hypertension have confirmed that treated hypertensive patients who smoke tobacco have a greater incidence of both stroke and coronary heart disease than equally treated hypertensive patients who do not smoke $(19,34)$. It is likely that the pressor effect of smoking (35) has been underestimated by the usual recommendation of avoiding smoking before measuring blood pressure. Repeated advice as to how to discontinue smoking is therefore of major importance, and will need to be coupled with particular efforts to prevent consequent weight gain. Intensive advice programmes, supported when necessary by pharmacological treatment of nicotine addiction, are more effective than haphazard admonitions.

Lipids. Since high serum cholesterol levels and diabetes also unfavourably influence the long-term prognosis of hypertensive persons, nutritional counselling and, when appropriate, drug treatment are indicated to control these risk factors. If dietary measures are to be successful, a careful follow-up should be instituted. Since increased physical activity is also likely to reduce the risk of cardiovascular disease, it is appropriate in mildly hypertensive patients.

\section{Oral contraceptive pills and hormonal replacement} therapy. Alternative methods of contraception should be considered for hypertensive women in place of estrogen-progesterone-containing oral contraceptives as these substances may raise the blood pressure as well as carry other cardiovascular risks (36).

Hormone replacement therapy is being increasingly used to prevent osteoporosis in postmenopausal women. There is evidence that replacement with estrogen alone reduces coronary risk (37), but the evidence is less for combined estrogen/progesterone therapy. Such combinations are generally recommended for women with an intact uterus to protect against uterine malignancy. There is no contraindication to the use of hormone replacement therapy in hypertension but blood pressure should be monitored more frequently, as it is not yet clear whether hypertensive responses may occur in some women.

It should be emphasized that the above-mentioned non-pharmacological interventions, whether or not they lower the blood pressure, can reduce the overall risk of cardiovascular disease and significantly contribute to global health care.

\section{Antihypertensive drugs}

Randomized trials of antihypertensive treatment have shown the benefits of lowering the blood pressure and although most of these trials have used diuretics, centrally acting drugs, vasodilators and/or beta-blockers, often in combination, there is no evidence so far to show that the benefits are due to any particular class of antihypertensive agents rather than to the lowering of blood pressure per se. Also in several of the recent trials of antihypertensive treatment in the elderly, as many as $60-70 \%$ of the patients on active treatment received a combination of two or more drugs, and the real evidence provided by these trials is about the benefit of lowering blood pressure.

Several classes of drugs can be recommended as first-line treatment of mild sustained hypertension. They may be listed, in order of proven benefit based on mortality-morbidity studies: (1) diuretics, (2) beta-blocking drugs, and (3) ACE inibitors, calcium antagonists, alpha-adrenoceptor blocking drugs.

While the average blood pressure fall induced in groups of patients by each of the different categories of drugs is similar, there are large variations in the reduction induced in the individual patient. The appropriate choice of a particular class of antihypertensive drugs for a patient may also be determined by the individual's other characteristics, since there are such extensive differences in the risk profile and in side-effects in different patients.

The choice of the initial drug therapy of an individual hypertensive patient is a challenge for the physician and should not be restricted, on theoretical or economic grounds, to any one or two of the various classes of drugs which have been tested so far, although it is also the physician's responsibility to give due consideration to the cost of drugs.

Finally, we should bear in mind that the real evidence of benefit (risk ratios for mortality, stroke, and coronary events) has been obtained largely over a relatively short time (of about 2.5 years to the terminating event). Such end-points may not be relevant for younger patients with hypertension, who may have decades of treatment ahead of them. For such patients a reduction in progression of cardiovascular lesions may be of greater relevance $(26,38)$.

\section{(a) Diuretics}

Diuretics have been widely used as first-line antihypertensive therapy and diuretic-based therapy has been shown to be clearly effective in the prevention of cardiovascular morbidity and mortality, especially fatal and non-fatal stroke (20). Particularly in large doses, diuretics may cause a variety of unwanted metabolic effects (principally potassium depletion and reduced glucose tolerance (39), ventricular 
ectopic beats, and impotence (19)), which can be reduced if the dose is kept as low as possible. Lowdose diuretics remain effective, not only in lowering elevated blood pressure, but in reducing cardiovascular morbidity and mortality, as shown in recent trials of antihypertensive therapy in the elderly (4-7). Diuretics are inexpensive. They are also particularly valuable as ancillary treatment to enhance the effectiveness of many other antihypertensive drugs. Combination of diuretics with potassium-sparing drugs or with ACE inhibitors may prevent potassium depletion.

\section{(b) Beta-adrenoceptor-blocking drugs}

Beta-blocking drugs are widely and effectively used to initiate treatment for mild hypertension. Betablocker-based antihypertensive therapy has been shown to reduce cardiovascular morbidity and mortality (20). Numerous beta-blocking drugs are available, some with cardioselective properties, others with partial agonist activity or with alpha-blocking or vasodilator properties. Although beta-blockers have been shown to prevent fatal and non-fatal coronary events in patients with a previous myocardial infarction (40), they have not been shown to have any consistent advantage over diuretics for the primary prevention of myocardial infarction.

Beta-blocking drugs are particularly useful in hypertensive patients with effort angina, tachyarrhythmias, or a past myocardial infarction. They should be avoided in patients with obstructive airways disease, heart failure, and peripheral vascular disease. They have limitations in patients with dyslipidaemia or reduced glucose tolerance (39), as well as in athletes or physically active subjects.

\section{(c) ACE inhibitors}

ACE inhibitors are effective in lowering blood pressure. They are generally well tolerated and have been shown not to exert untoward effects on serum lipids and on glucose homoeostasis (39). Possible adverse effects include persistent cough and, rarely, angiooedema. In patients with renovascular disease, deterioration in renal function has been reported. ACE inhibitors should be avoided in women considering child-bearing and are definitely contraindicated in the second half of pregnancy, since they may increase fetal and neonatal death (41). Their safety profile is otherwise good.

ACE inhibitors have been shown to reduce cardiovascular mortality and morbidity, including coronary events (42-45), in patients with congestive heart failure and after a myocardial infarction in patients with a reduced ejection fraction. In these patients a reduction of left ventricular dilatation has also been reported (44). The ability of the ACE inhibitors to reduce cardiovascular events in hypertension has not yet been proven in controlled clinical trials.

\section{(d) Calcium antagonists}

There are three major classes of calcium antagonists with different characteristics (phenylalkylamine, dihydropyridine and benzothiazepine derivatives), but all are effective in lowering blood pressure.

Troublesome side-effects may include initial tachycardia, headache, flushing (especially with fastacting dihydropyridines), ankle oedema and (with phenylalkylamine derivatives), and constipation. With few exceptions, they do not have undesirable metabolic effects (39) and their safety profile in hypertension appears good. However, the safety of dihydropyridines in early pregnancy has not been fully established. In patients with atherosclerotic arterial disease the calcium antagonists may reduce the development of new plaques (46), but studies with fast-acting short-duration dihydropyridines have been discouraging in providing evidence of secondary prevention of ischaemic heart disease $(47,48)$. On the other hand, verapamil and diltiazem have been reported to reduce morbidity and mortality in post-myocardial infarction patients, provided that heart failure or left ventricular dysfunction are absent (47). As with all recent classes of antihypertensive agents, the calcium antagonists' ability to reduce cardiovascular events in hypertension has not yet been proven in controlled clinical trials.

\section{(e) Alpha-adrenoceptor-blocking drugs}

Alpha-adrenoceptor-blocking drugs effectively reduce blood pressure and have limited side-effects. Their dose should be carefully titrated, however, in order to avoid postural hypotension, a complication particularly undesirable in the elderly. A favourable aspect of this class of drugs is a potential beneficial effect on lipid and glucose homoeostasis (39). As with all newer classes of antihypertensive agents, the alpha-blockers' ability to reduce morbidity and mortality has not been proven in controlled clinical trials.

\section{(f) Other classes}

Centrally acting drugs are also effective antihypertensive agents and have been used for many years, mostly in association with diuretics. Several controlled clinical trials have proved their ability in antihypertensive therapy to reduce cardiovascular events (20). In particular, methyldopa remains an important, well-validated agent to treat effectively hypertension in pregnancy (49). The side-effect profile of centrally acting drugs, however, is less favourable than that for the antihypertensive agents previously men- 
tioned. If consideration of cost-effectiveness should favour the use of centrally acting drugs, such as reserpine, in low-income populations, it is recommended that they be used in much lower doses than commonly prescribed in earlier years.

Direct vasodilators, such as hydralazine and minoxidil, are also quite effective in lowering blood pressure, but some of their side-effects (tachycardia, headache, and sodium and water retention) make it difficult to use them as monotherapy.

\section{(g) Combination of drugs}

If monotherapy with a compound of any of the five major pharmacological classes mentioned above has been found ineffective in lowering blood pressure in a given patient, it is reasonable to substitute the first drug with a compound belonging to a different class. If a single drug has been partly effective it may be preferable to add a small dose of a second drug rather than to increase the dose of the first. Effective combinations utilize compounds from different drug groups. This permits the addition of different primary actions while minimizing the homoeostatic compensations that limit the fall in pressure. Combination therapy also minimizes side-effects by encouraging the use of drugs in low doses.

An additive effect has been shown when combining:

- a diuretic with a beta-blocker or an ACE inhibitor or an alpha-blocker;

- a beta-blocker with an alpha-blocker or a dihydropyridine calcium antagonist; and

- an ACE inhibitor with a calcium antagonist.

In order to achieve the full goal of antihypertensive treatment in all hypertensive patients (i.e., the maximum tolerated reduction in blood pressure), two-drug and sometimes three-drug combinations may frequently be required.

For reasons of convenience, cost and increased patient compliance, preparations that combine two drugs in a single tablet or capsule may be appropriate for many hypertensive patients, once the need and dose for the constituent drugs have been established.

\section{Follow-up}

During the stabilization period of treatment, patients need to be seen at regular intervals until the blood pressure levels are satisfactorily controlled. The main task of doctors during follow-up is to ensure that the target systolic and diastolic blood pressure is reached and maintained and that other risk factors are controlled. Gradual and careful lowering of blood pressure will minimize the side-effects and compli- cations, and will improve compliance. Sometimes telling a patient that he or she has hypertension ("labelling") may be followed by anxiety or mood changes. Additional support (e.g., reassurance about the prognosis, stress on the ability to lead normal active lives, and explanation of any new symptoms that may appear) is therefore particularly important. Self-measurement of blood pressure may be helpful to ensure compliance. After stabilization of blood pressure, follow-up visits at 3-6 month intervals may be adequate.

During each visit the blood pressure should be measured and side-effects monitored, including a careful assessment of the patient's quality of life. Whenever possible, close contact with the patient's family is helpful. During follow-up visits, nonpharmacological measures (particularly cessation of smoking, control of serum cholesterol and weight, moderation of alcohol intake, and institution of physical exercise) should be reinforced. Pharmacological therapy should be reconfirmed or readjusted. In cases resistant to therapy further etiological investigation should be performed. Depending on the drugs used, appropriate laboratory investigations should be performed at regular intervals.

As a rule, antihypertensive therapy should be maintained indefinitely. Cessation of therapy in patients who had been correctly diagnosed as hypertensives (see criteria above and flow-chart) is usually followed-sooner or later-by return of blood pressure to pretreatment levels. Nevertheless, after prolonged blood pressure control it may be possible to attempt a careful progressive reduction in the dose or number of drugs used, especially in patients strictly observing non-drug treatment. Attempts to step down the treatment should be accompanied, however, by careful, continued supervision of blood pressure.

A hypertensive patient is typically treated for several decades, and during that period major changes in the available drugs occur and new practical issues arise. It is likely that under the influence of pharmacological progress, as well as habits, advertisements or economical considerations, a patient's treatment will undergo multiple changes. It is in the interest of every patient to carefully document all the drugs taken over many years, in conjunction with a careful analysis of their efficacy, clinical tolerability and biochemical effects. It is also the responsibility of both doctors and health services to make the history of a treated hypertensive patient easily available.

\section{Cost-effectiveness}

The cost-effectiveness of treating hypertension varies markedly with the degree of cardiovascular risk in 
various sủbgroups of patients with hypertension. Cost-effectiveness is more apparent in elderly subjects and in patients with previous cardiovascular disease, in whom the benefit is realized within a shorter time span, than it is in younger patients, in whom the benefit is delayed for many years during which the costs of management accumulate. On the other hand, the costs of treating cardiovascular complications of hypertension such as stroke make the treatment of hypertension a very cost-effective process in high-risk groups, in whom the incidence of stroke is reduced by over $40 \%$.

While the costs of relatively expensive diagnostic procedures and drugs are readily justified in highrisk groups, the practising physician should give careful consideration to the costs associated with the investigation and treatment of patients with mild hypertension without associated risk factors. Primary prevention of hypertension and strategies based on changing the lifestyle of the whole population may offer the most cost-effective means of reducing the morbidity and mortality associated with mild hypertension. The effectiveness of these approaches in primary prevention requires further validation.

\section{Further research}

Many issues require resolution and some are being addressed by major studies already in progress. The question of goal blood pressures and the "J curve" is being addressed by the HOT study (33). Evidence for reduction of morbidity and mortality by the newer classes of antihypertensive drugs is clearly needed. This question is currently being addressed by the STOP-II (50) and the Syst-Eur (51) studies in the elderly. A major study in middle-aged subjects comparing the benefits of the newer agents with those provided by diuretics and beta-blockers may also be desirable.

In young and middle-aged subjects with mild hypertension, in whom the short-term incidence of cardiovascular events is rather low, trials monitoring progression/regression of organ damage (such as left ventricular hypertrophy, carotid artery wall mediaintima thickness and plaques, proteinuria and microalbuminuria) may also provide relevant information on the comparative effectiveness of various classes of antihypertensive agents. A related issue is whether the reversal of organ damage, in particular left ventricular hypertrophy, is accompanied by commensurate reduction in cardiovascular risk. It would also be important to investigate whether combining antihypertensive and antiplatelet therapies can further reduce cardiovascular events in hypertensive patients (as is being studied in the HOT trial (33)), and whether combining antihypertensive and lipid-lower- ing treatments can help to reduce the progression of atherosclerosis. Another important issue concerns the potential benefit of lowering blood pressure in subjects with blood pressures lower than $140 / 90 \mathrm{mmHg}$ but with other factors that markedly increase the risk (diabetes, previous transient ischaemic attack, nephropathies, etc.). Methods for optimizing the choice of antihypertensive drugs for each individual patient need to be improved, and research on the effectiveness of different methods of hypertensive management should be promoted in different countries and populations.

More data are clearly needed on the prognostic significance of blood pressure values obtained by ambulatory blood-pressure monitoring or by selfmeasurements taken at home or at work. The quantification and the prognostic significance of "white coat" hypertension should be addressed.

Finally, developments in genetic research may help to clarify further which hypertensive patients are at particular risk of developing cardiovascular disease and events, and indicate the subjects in greatest need of preventive therapy.

\section{References}

1. MacMahon, S. et al. Blood pressure, stroke and coronary heart disease. Part 1. Prolonged differences in blood pressure: prospective observational studies corrected for the regression dilution bias. Lancet, 335: 765-774 (1990).

2. Evans, J.G. \& Rose, G. Hypertension. Br. med. bull., 27: 765-774 (1990).

3. Kannel, W.B. Hypertension and the risk of cardiovascular disease. In: Laragh, J.H. \& Brenner, B.M., ed. Hypertension: pathophysiology, diagnosis, and management. New York, Raven Press, 1990, pp. 101-117.

4. Amery, A. et al. Mortality and morbidity results from the European Working Party on High Blood Pressure in the Elderly trial. Lancet, 1: 1349-1354 (1985).

5. SHEP Cooperative Research Group. Prevention of stroke by antihypertensive drug treatment in older persons with isolated systolic hypertension. J. Amer. Med. Assoc., 265: 3255-3264 (1991).

6. Dahlöf, B. et al. Morbidity and mortality in the Swedish Trial in Old Patients with Hypertension (STOP-Hypertension). Lancet, 338: 1281-1285 (1991).

7. MRC Working Party. Medical Research Council trial on treatment of hypertension in older adults: principal results. Br. med. j., 304: 405-412 (1992).

8. MRC Working Party. Stroke and coronary heart disease in mild hypertension: risk factors and the value of treatment. $\mathrm{Br}$. med. j., 296: 1565-1570 (1988).

9. MRC Working Party on Mild to Moderate Hypertension. The MRC Mild Hypertension trial: some subgroup results. In: Strasser, T. \& Ganten, D., ed. 
Mild hypertension: from drug trials to practice. New York, Raven Press, 1987, pp. 9-20.

10. Mancia, G. et al. Alerting reaction and rise in blood pressure during measurement by physician and nurse. Hypertension, 9: 209-215 (1987).

11. Julius, $\mathbf{S}$. et al. White coat hypertension: a followup. Clin. exper. hypertens., A14: 45-53 (1992).

12. Mancia, G. Ambulatory blood pressure monitoring: research and clinical applications. J. hypertens., 8 (suppl. 7): S1-S13 (1990).

13. O'Brien, E. et al. The British Hypertension Society protocol for the evaluation of automated and semiautomated blood-pressure-measuring devices with special reference to ambulatory systems. J. hypertens., 8: 607-619 (1990).

14. Sega, R. et al. Ambulatory and home blood pressure normality: the PAMELA Study. Abstr. 6th European Meeting on Hypertension, Milan, 4-7 June 1993, Abstract No. 694.

15. Chatellier, G. et al. Decision to treat mild hypertension after assessment by ambulatory monitoring and World Health Organization recommendations. $\mathrm{Br}$. med. j., 305: $1062-1066$ (1992).

16. Zanchetti, A. What blood pressure level should be treated? In: Laragh, J.H. \& Brenner, B.M., ed. Hypertension: pathophysiology, diagnosis, and management. New York, Raven Press, 1990, pp. 1967-1983.

17. Rose, G.A. Hypertension in the community. In: Bulpitt, C.J., ed. Epidemiology of hypertension (Handbook of hypertension series, Vol. 6), pp. 1-14, Amsterdam, Elsevier, 1985.

18. Report by the Management Committee. The Australian Therapeutic Trial in Mild Hypertension: untreated mild hypertension. Lancet, 1: 185-191 (1982).

19. Medical Research Council Working Party. MRC trial of treatment of mild hypertension: principal results. Br. med. j., 291: 97-104 (1985).

20. Collins, R. et al. Blood pressure, stroke, and coronary heart disease. Part 2. Short-term reductions in blood pressure: overview of randomised drug trials in their epidemiological context. Lancet, 335: 827-838 (1990).

21. WHO/ISH Guidelines Committee. Prevention of hypertension and associated cardiovascular disease: a 1991 statement. Clin. exper. hypertens., A14: 333-341 (1992).

22. Kannel, W.B. et al. Perspectives on systolic hypertension: the Framingham study. Circulation, 61: 1179-1182 (1986).

23. Joffres, M.R. et al. for the Canadian Heart Health Surveys Research Group. Prevalence, control and awareness of high blood pressure among Canadian adults. Can. Med. Ass. j., 146: 1997-2005 (1992).

24. Joint National Committee on Detection, Evaluation, and Treatment of High Blood Pressure. The Fifth Report of the Joint National Committee on Detection, Evaluation, and Treatment of High Blood Pressure (JNCV). Arch. int. med., 153: 154-183 (1993).

25. WHO Technical Report Series, No. 628, 1978 (Arterial hypertension: report of a WHO Expert Committee).
26. Zanchetti, A. Goals of antihypertensive treatment: prevention of cardiovascular events and prevention of organ damage. Blood pressure, 1: 205-211 (1992).

27. Levy, D. et al. Prognostic implications of echocardiographically determined left ventricular mass in the Framingham Heart Study. New Engl. j. med., 322: $1561-1566$ (1990).

28. Koren, M.J. et al. Relation of left ventricular mass and geometry to morbidity and mortality in uncomplicated essential hypertension. Ann. intern. med., 114: 345-352 (1991).

29. Shulman, N.B. et al. Prognostic value of serum creatinine and effect of treatment of hypertension on renal function. Hypertension, 13 (suppl. 1): 80-93 (1989).

30. Ruilope, L.M. Proteinuria and renal function. High blood press., 2(suppl. 1): 50-53 (1993).

31. Parving, H.-M. \& Hommel, E. Prognosis in diabetic nephropathy. Br. med. j., 299: 230-233 (1989).

32. Cruickshank, J.M. et al. Benefits and potential harm of lowering high blood pressure. Lancet, 1: 581-584 (1987).

33. The HOT Study Group. The Hypertension Optimal Treatment Study (The HOT-Study). Blood pressure, 2: $62-68$ (1993).

34. Langford, H.G. et al. All-cause mortality in the Hypertension Detection and Follow-up Program: findings in the whole cohort and for persons with less severe hypertension, with and without other traits related to risk of mortality. Progr. cardiovasc. dis., 29(suppl. 1): 29-54 (1986).

35. Mancia, G. et al. Cardiovascular effects of smoking. Clin. exper. hypertens., A12: 917-929 (1990).

36. Layde, P.M. et al. Further analyses of mortality in oral contraceptive users (Royal College of General Practitioners' Oral Contraception Study). Lancet, 1: 541-546 (1981).

37. Wren, B.G. \& Routledge, D.A. Blood pressure changes: oestrogens in climacteric women. Med. $j$. Australia, 2: 528-531 (1981).

38. Zanchetti, A. et al. Evaluation of organ damage in hypertension. Consensus document. High blood pressure, 2(suppl. 1): 92-104 (1993).

39. Lithell, H. Insulin resistance and cardiovascular drugs. Clin. exper. hypertens., A14: 151-162 (1992).

40. Yusuf, S. et al. Beta-blockade during and after myocardial infarction. An overview of the randomized trials. Progr. cardiovasc. dis., 27: 335-371 (1985).

41. Kreft-Jais, C. et al. Angiotensin-converting enzyme inhibition with captopril in human pregnancy. $J$. hypertens., 5(suppl. 5): S553-S554 (1987).

42. The SOLVD Investigators. Effect of enalapril on survival in patients with reduced left ventricular ejection fractions and congestive heart failure. New Engl. j. med., 325: 293-301 (1991).

43. The SOLVD Investigators. Effect of enalapril on mortality and the development of heart failure in asymptomatic patients with reduced left ventricular ejection fraction. New Engl. j. med., 327: 685-691 (1992).

44. Pfeffer, M.A. et al. Effects of captopril on mortality and morbidity in patients with left ventricular dys- 
function after myocardial infarction: results of the Survival and Ventricular Enlargement trial. New Engl. j. med., 327: 669-677 (1992).

45. Yusuf, $S$. et al. Effect on enalapril of myocardial infarction and unstable angina in patients with low ejection fractions. Lancet, 340: 1173-1178 (1992).

46. Zanchetti, A. The antiatherogenic effects of antihypertensive drugs: experimental and clinical evidence. Clin. exper. hypertens., A14: 307-331 (1992).

47. Yusuf, $S$. et al. Update of effects of calcium antagonists in myocardial infarction or angina in light of the Second Danish Verapamil Infarction Trial (DAVIT-II) and other recent studies. Am. j. cardiol., 67: 1295-1297 (1991).
48. Goldbourt, U. et al. for the SPRINT Study Group. Early administration of nifedipine in suspected acute myocardial infarction. Arch. intern. med., 153: 345-353 (1993).

49. Reid, J.L. \& Elliott, H.L. Methyldopa. In: Doyle, A.E., ed. Clinical pharmacology of antihypertensive drugs (Handbook of hypertension series, Vol. 5), pp. 92-112, Amsterdam, Elsevier, 1984.

50. Dahlöf, B. et al. STOP-hypertension 2. Abstr. 6th European Meeting on Hypertension, Milan, 4-7 June 1993, Abstract No. 165.

51. Amery, A. et al. Syst-Eur. A multicenter trial on the treatment of isolated systolic hypertension in the elderly: objectives, protocol and organization. Aging, 3: 287-302 (1991). 